\title{
OTTI WILMANNS
}

\section{Das Wechselspiel von Beobachtung, Fragestellung und Folgerung}

Zur Didaktik und Methodik botanischer Exkursionen 
Sonderdruck: Verhandlungen der Gesellschaft für Ökologie, Göttingen 1976.

\title{
DAS WECHSELSPIEL VON BEOBACHTUNG, FRAGESTELLUNG UND FOLGERUNG: ZUR DIDAKTIK UND METHODIK BOTANISCHER EXKUR- SIONEN
}

\author{
O WILMANNS
}

\begin{abstract}
Today it is necessary to promote the basic knowledge of the ecological connections in the direct environment of the students and their ability to put it into practice. One way towards this aim is a more intensive training during botanical excursions in the following sense: 1. Activation of the students' capacity to observe, to combine and to draw conclusions from the observed facts and theoretical background information; 2. elucidation of ecological and historical connections; 3. emphasis on connections with other disciplines. This is demonstrated by some situations during excursions and a list of concrete examples for general problems.
\end{abstract}

Seit mindestens 10-20 Jahren ist zu beobachten, daß unsere angehenden Biologen - von Ausnahmen abgesehen - ein äußerst fragmentarisches Grundwissen über Pflanzen und Tiere ihrer Heimat besitzen und ihre Beziehung zur freien Natúr sich nicht von der des Laien unterscheidet, d.h. sich allenfalls auf ästhetisches Wohlgefallen beschränkt. Dies ist längst bekannt; - beklagt aber wird es - wiederum von Ausnahmen abgesehen - erst seit wenigen Jahren. Heute ist man jedenfalls nicht mehr gesonnen, es hinzunehmen, daß ein Staatsexamenskandidat mit Hauptfach Biologie zwar spontan auf einem Bild einen Darwinfinken erkennt und zu benennen weiß, nicht aber Kohlmeise, Bläßhuhn oder Grille. Ein entscheidender Grund, ja wohl der Auslöser des Wandels ist die Umweltproblematik, die gerade zu dem Zeitpunkt ins öffentliche Bewußtsein rückte, als die Faszination der Molekularbiologie nachzulassen begann und das durch sie so ungeheuer erweiterte neue Weltbild zum Allgemeingut wurde.

Was also tun? Eine Förderung rein theoretischer umweltbezogener Kenntnisse, etwa im Lehrplan der Oberstufe und an der Universität, ist gewiß gut und notwendig; aber es ist doch deutlich, daß hierbei ein wesentlicher Punkt fehlt: die unmittelbare Beziehung zum Organismus und zur Lebensgemeinschaft im Freiland, zum ganz konkreten Fall und damit auch zum praxisnahen ökologischen Denken. Das Prinzip der Nahrungskette zu kennen, um ein Beispiel zu geben, ist gewiß wesentlich und auch leicht erreichbar. Im konkreten Einzelfall aber kann es heißen: „Wo haben jene roten, acht-beinigen, saugenden Tierchen an unserm Apfelbaum ihren Platz darin? Wer frißt sie? Warum haben sie überhandgenommen und sind zum Schädling geworden? "

Einen Weg - unter anderen - hier allmählich Wandel zu schaffen, bietet die klassische Lehrveranstaltung der Exkursion. Das heißt allerdings nicht, daß sie einfach in der tradierten Weise, d.h. mit dem Hauptziel der Rezipierung floris- 
tischen Wissens, nur allenfalls mit höherer Stundenzahl und schärferem Abprüfen des Stoffes, durchgeführt werden sollte. Vielmehr scheinen mir zwei Dinge notwendig: Es sind 1. größere geistige Aktivität der Lernenden und 2. stärkere Verdeutlichung der ökologischen und historischen Zusammenhänge und der Beziehungen zu andern Fachrichtungen. Gerade der Wunsch vieler Studenten nach gründlichen Kenntnissen in der Freilandbiologie hat aber auch das alte Problem erneut aufgeworfen: „Wie werden wir der Fülle Herr? Wo finden wir einen Ariadnefaden?" oder vom Lehrer aus formuliert: „Wie überwinden wir die anfängliche Fntmutigung, und wie bringen wir den Lernenden so weit, daß er sich hinfort selbständig weiterhelfen kann? Wie lernt er, seine belebte Umgebung als jeweils spezifisches Beziehungsgefüge zu erkennen, als historisch gewordenes Öko-System?" Dabei darf man eines nicht übersehen: Es handelt sich hierbei nicht um bisher ganz unbekannte gedankliche Ansätze oder um uns bisher unzugängliche Dimensionen (wie bei dem wissenschaftlichen Neuland der Molekularbiologie und der submikroskopischen Zellstrukturforschung); vielmehr befinden wir uns glcichsam in der Situation des Goethe'schen Schauspieldirektors, der da fragend ausruft:

„Wie machen wir's, daß alles frisch und neu und mit Bedeutung auch gefällig sei?"

Es handelt sich also um ein didaktisch-methodisches Problem, zu welchem ich einen kleinen Beitrag leisten möchte, dies auf Grund persönlicher Erfahrung als Lernende wie auch als Lehrende.

Drei - einander nicht ausschließende, sondern ergänzende - Möglichkeiten seien vorweg theoretisch formuliert und später an Exkursionssituationen veranschaulicht:

1. Die Exkursion wird ausdrücklich unter ein bestimmtes Generalthema gestellt (oder unter einige wenige solcher Arbeitsziele). (Hierzu vergleiche man das anregende Buch von M. MOOR: Vegetationskunde der Umgebung von Basel, 1962.) 2. Man ordnet möglıchst viele Details jeweils in ein umfassendes theoretisches Gerüst, in ein bekanntes Begriffssystem ein.

3. Es wird die Fähigkeit zu Beobachtung und selbständigem Schlußfolgern gefördert. (Übrigens: Die eine Teilwahrheit enthaltende und ein wenig abwertende Behauptung ,Man sieht nur, was man weiß" hat hier keinen Platz; schon dic Tatsache, daß ein Lernender wiederentdeckt, was ihm zuvor theoretisch vermittelt worden war, ist nicht selbstverständlich.)

Für das konkrete Vorgehen ist natürlich jeweils der vorhandene Kenntnisstand ausschlaggebend; er macht eine stufenspezifische Differenzierung notwendig. Auch sind geowissenschaftliche Grundkenntnisse unabdingbar, wenn Analyse und Synthese, Induktion und Deduktion bewältigt werden sollen.

Einige Beispicle für Exkursionsarbeit im Gelände (A) sollen das Gesagte illustrieren. Diese Reihe möge gewiß nicht als Muster einer bestimmten Exkursion aufgefaßt werden; vielmehr sind es einzelne Passagen, die in den üblichen, morphologisch-floristisch ausgerichteten Ablauf eingeschaltet werden können. Es bedarf kaum der Erwähnung, daß ein Frage - Antwort - Spiel auch nie in solcher ,Glätte“ vonstatten geht. Fragen können sowohl vom Lehrenden wie 
von den Schülern oder Studenten stammen; dic Antwort wird nicht immer in der erwarteten Weise erfolgen. Vorgestellt werden vielmehr Idcalfälle, welche die methodische Zielrichtung verdeutlichen sollen. - Als Gebiet wurde der für meine Universität Freiburg in doppeltem Sinne naheliegende Kaiscrstuhl gewählt. Diese Zusammenstellung (A) erwies sich als derart umfangreich, daß ich hier auf eine exemplarische Behandlung anderer Gebiete verzichten muß und daß überdies der knappe mündliche Bericht nur als Erläuterung für das ausführlicher schriftlich Dargestellte dienen kann. Anschließend soll der Versuch gewagt werden, eine - sicher nicht vollständige und mehr zur Anregung bestimmte - Übersicht (B) zu geben über ökologische Prinzipien und Problemkreise, zu denen man auf Exkursionen Einzelfälle ableiten kann; oder umgekehrt: Beobachtbares, das im theoretischen Unterricht als Erlebnis aus eigener Anschauung und damit lebendiges Beispiel dienen kann.

\section{A. Beispiel Kaiserstuhl}

Im Voraus gebotene Charakteristik: kollin-submontanes Gebiet $(200-557 \mathrm{~m}$ $\mathrm{NN}$ ); Klimadaten für Oberrotweil im langjähr. Mittel: $9,9^{\circ} \mathrm{C}$ Jahresmitteltemperatur, $682 \mathrm{~mm}$ Niederschlag, 50,3 Sommertage, 77,7 Frosttage (vgl. mit andern Gebieten!). Ausgeprägtes Relief über meist vulkanitischem Untergrund mit Lößmantel. Knapp zur Hälfte Rebgelände; berühmte Trespenrasen; mannigfache Wälder, davon wenig Staatswald. Reich an submediterranen und kontinentalen Pflanzen- und Tierarten. (Hinweis: Abschnitt I bis III beziehen sich auf die Gesichtspunkte: Überblick - Rebgelände - Wälder. Ein Abschnitt über Trespenrasen wurde aus Platzgründen ausgespart. Pfeile bezeichnen die anzustrebende Dialogrichtung.) 
I. Úberblick (an Aussichtspunkt; dabei soll jedenfalls eine geologische Karte verfügbar sein; günstig ist auch eine Vegetations- und Schutzgebietskarte)

\begin{abstract}
Ausgangsbeobachtung bzw. mitgeteilte, u.U. bekannte Tatsache

daraus hergeleitete Frage bzw. lenkende Zusatzfrage
\end{abstract}

\section{Antwort}

entw. aus weiterer Beobachtung

und/oder als (deduktive) Folgerung

und/oder als (Arbeits-) Hypothese
Generalisierung,

Hinweis auf verwandte Sachgebie-

Das Gebiet ist reich an wissenschaft-

lich wertvollen und beliebten selte-

nen Arten und Gesellschaften

Welche Gründe gibt es dafür?

Für Mitteleuropa ungewöhnliche Standortsbedingungen; dies kann klimatisch und/oder edaphisch (geologisch) und/oder durch die Bewirtschaftung begründet sein. Vermutlich Xerothermie der

In der Tat zeichnen sich die entStandorte ausgeprägt.

sprechenden Standorte meist durch ungewöhnliche Wärme u. Trockenheit aus; 4 der 5 Naturschutzgebiete (vgl. Karte) liegen an steilen S-bis W-Hängen im zentralen od. westl. Teil des Gebirges.

s. $\longrightarrow$

Wie erklärt sich die Xerothermie als ökologische Eigenart des Kaiserstuhls? a) Regenschatten der Vogesen im W; infolge Aufstieg von Luftmassen am Schwarzwaldrand im $\mathrm{O}$ ist $\mathrm{Zu}$ nahme des Niederschlags gegen $O$ zu erwarten;

b) geringe Meereshöhe, freier Zutritt von SW-Winden durch Burgundische Pforte haben zur Folge milde Winter und warme Sommer; c) steiles Relief inf. harter Gesteine im Untergrund; daher kleiner Mikroklima

Einstrahl. winkel an S-Hängen

(Cosinus-Ges.);

d) folgl. hohe Erwärmung; Steil-

heit hat Flachgründigkeit zur 
Prägen sich diese Verhältnisse

Pflanze Trockenheit.

auch im Anbau aus?

Welche Hauptnutzungen gibt es? $\rightarrow$ a) Reben ca. 1/2 der Fläche;

b) Wald ca. 1/5 der Fläche;

c) Obst als Plantagen u. als Streuobstbau sowie Acker (lokal)

Zeigen diese Nutzungstypen eine gesetzmäßige räumliche Anordnung?

d) Wiesen meist ungemäht (lokal).

a) Rebflur: Hänge; teilw. junge Anrisse, also Areal erweitert;

b) Wald: Kammlagen;

c) Obst u. Äcker: Täler;

d) Wiesen: Hänge des ZentralKaiserstuhls, teilweise in

Vgl. geolog. Karte:

Kämme ohne Lößbedeckung diese Anordnung?

\section{Tälchen.}

a) Rebanbau meidet kaltluftgefährdete Lagen; hohe und nach hinten geneigte Terrassen ungünstig; Fehlen in höchsten Lagen auf Kälte und Wind und/ oder Flachgründigkeit zurückzuführen

b) Wald: am wenigsten arbeitsaufwendig, daher in Ortsferne; dazu klimatisch u. edaphisch ungünstige Standorte besiedelnd.

c) Obst: Täler feucht, daher für Reben ungünstig (Kälte, Bodenverdichtung).

d) Wiesen: steile u. flachgrund. Standorte; sicher nie sehr er-

Warum dann nicht auch hier

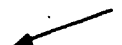
stattdessen Wald? Vgl. hierzu die Gemarkungszugehörigkeit der entspr. Wiesenareale!
Man bedenke auch fundamentele Abweichungen vom Hüttenklima!

Kaltluftentsteh

-fluß, -stau.

Standortgemäße Anbauplanung (z.T. gesetzl. Regelungen).
Nächste Orte bzw. Gemarkungen

sind Schelingen und

Oberbergen.
Lage im Innern des Kaiserstuhls, daher kein ausreichender Wiesenbesitz in $\mathrm{der}^{\circ}$ Ebene. 
II. Pflanzengesellschaften im Rebgelande: Eigentliche Rebflächen und ihre Unkräuter; die Besprechung des Böschungsbewuchses und des Vegetationsmosaiks der Hohlwege ist lohnend, doch muß an dieser Stelle auf sie verzichtet werden.

Ausgangsbeobachtung

bzw. mitgeteilte, u.U. bekannte

Tatsache

daraus hergeleitete Frage

bzw. lenkende Zusatzfrage

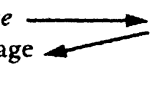

Die Stammform der Kulturrebe,

Vitis sylvestris, ist eine Kletter-

pflanze des Mantels von Auwaldern.

Ihre Eigenschaften bestimmen die

Methoden des Rebbaus: Erziehung

an Drähten, Schnitt auf guten

Lichtgenuß, häufige, aber oberflàch-

liche Bodenlockerung, gute Dun-

gung.

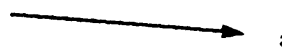

a) Welche Lianen-Eigenschaften sind zu beobachten?

b) Weshalb wird nur bis etwa $10 \mathrm{~cm}$ tief gehackt und gepflügt bei reichlicher

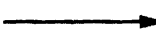

\section{Antwort}

entw. aus weiterer Beobacbtung und/oder als (deduktive) Folgerung und/oder als (Arbeits-) Hypothese
Generalısierung

Hinweis auf verwandte Sachgebiete
Düngung?

a) Ranken, die sich nach Stellung als Sprosshomologe erweisen; weitlumige Tracheen, notwenwendig für ausreichende Wasserleitung, da nur geringer Holzquerschnitt zur Festigung nötig ist.

b) Entsprechende Auwaldböden sind gut durchlüftet und nährstoffreich. Ein Teil des Wurzelsystems tiefgehend, ein Teil oberflächlich in etwa $15 \mathrm{~cm}$ ergrabbar.

Welchen Sinn mag der recht große Zeilenabstand von 1,50 oder $1,80 \mathrm{~m}$ haben?

Ausreichender Arbeitsraum für Maschinen - Durchlichtung Windzug bewirkt rascheres Abtrocknen nasser Blätter $u$. verringert dadurch Pilzgefahr.

\section{Homologiebegriff; andere Klettermodi}

Wasserleitung im Sproß. 
Zuweilen findet man Zwischensaat von Raps oder Senf.

Wozu dient diese?

-

Man findet eine Reihe von Unkrautarten, die von Äckern, insbes. Hackfruchtäckern, bekannt sind; dazu einige rebspezifische.

Zur ersten Gruppe gehören Stellaria media, Lamium purpureum u. Convolvulus arvensis; zur zweiten Allium vineale, Muscari racemosum, Ornithogalum spec.
(Von Fortgeschrittenen kann aufgrund früherer Beobachtung oder Lehre eine Aufzählung solcher Arten erwartet werden.)

Wodurch kann diese Artenkombination bestimmt sein? Welche Standorts- und Bewirtschaftungseigenarten zeichen den Rebberg aus?

Entscheidene und für die einzelnen Arten in verschiedenem Maße wirksame Faktoren sind diskutabel:

gute Düngung - hohe Temperaturen der Luft und des Bodens - häufiges

Hacken im Sommer (, Rühren") -

Reben als Dauerkultur, d.h. nur

etwa alle 20 Jahre tiefgehende Bo-

denbearbeitung.
Bestimmend für die Rebspezifitat höchstwahrscheinlich die Bewirtschaftung.
Laßt sich eine morphologische oder physiologische Eigenschaft der genannten Arten finden, die dies zu erklären erlaubt?

Hinweis auf Vermehrungsweise und Vegetationsperiode; Studium der unterirdischen Organe.
Wirkung von Pflanzenwuchs auf den Boden.
Unkrautbekämpfung$$
\text { hoher Wasserverbrauch }
$$

der Unterfrucht

lockerung möglich. 
Die unspezifischen Unkrauter sind entweder kurzlebige Thero-

phyten oder (regenerationsfähige) Dauerunkräuter, die spezifischen jedoch Geophyten, die im März/

April blühen und deren Zwiebeln in 5-10-20 cm Tiefe liegen bzw. sich dort einregulieren. Durch Hacken auch Ausbreitung von Tochterzwiebeln.

Man sieht im Fruhling einzelne ungepflügte Parzellen mit fußhohen Decken von Vogelmiere.

$\longrightarrow$ Könnte dies einem bestimmten Zweck dienen?

$\longrightarrow$

Erhaltung gegen oberflächliche, unproduktive Wasserverluste, gegen Verkrustung und Erosion, bei Mulchen Humuslieferung (früher als Futter gemäht).
Ausbreitungsstrategien von Pflanzen.

Das allgemeine Problem: Unkraut oder Wildkraut?

\section{Wälder}

Ausgangsbeobacbtung bzw. mitgeteilte, u.U. bekannte Tatsacbe

daraus hergeleitete Frage bzw. lenkende Zusatzfrage

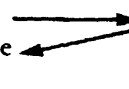

Antwort

entw. aus weiterer Beobachtung und/oder als (deduktive) Folgerung und/oder als (Arbeits-) Hypotbese

\section{Generalisierung}

Hinweis auf verwand te Sachgebiete

Man beobachtet bei geschickter

Routenführung abwechslungs-

reiche Waldbilder.

Woran kann das liegen?

Anweisung:

Um dies zu klären, muß eine
Es könnte

a) auf Holzartenzahl, die auf raschen Standortwechsel weist, und/oder

b) auf versch. Struktur der Wälder beruhen, die durch verschiedenartige Bewirtschaftung $u$. versch. Alter zustandekommt. 
Gruppe von Studenten auf Kriterien für a), eine andere auf solche für b) achten.

Zusatzaufgabe c): Wie verhält sich die Buche als von Natur aus dominierende Holzart?

Beispiele für a)

Umschlag der Artenkombination z.B. von Kuppen zu Mulden, von Essexit- zu Löß-Substrat;

Beispiele für b) stellenweise reichlich Stockausschläge (bes. Linde, Hasel, Elsbeere) als Folge von Nieder- $u$. Mittelwald-Wirtschaft;

Ausprägung geschich tlicher

Beobachtungen zu c)

Buche stellenweise dominierend, dann wieder fehlend trotz nichtextremen Standorts, of $t$ mit scharfer Bestandsgrenze, also anthropogen; zuweilen im Unterwuchs reichlicher als in der Baumschicht.

Wie könnte man nachweisen, daß

Besprechung von Bodenprofilen.

die Buche in der (potentiellen)

natürlichen Vegetation eine größe-

re Rolle spielen würde?

Man könnte

a) alte und rezente soziologische Aufnahmen vergleichen;

b) den Altersaufbau der Bestände testen (Buche im Unterstand würde für Nachlassen der Hemmung sprechen).

Auf Schlagflächen trotz relativ

xerischen Klimas ausgesprochene

Feuchtezeiger wie Cirsium palustre und Epilobium hirsitum zu

beobachten.

Wie ist das zu erklären?

Welche Ursache denkbar?

Wegen Fehlens des Kronendaches geringere Interzeption, damit 30-

Beziehungen zwischen den Gliedern $50 \%$ mehr Niederschlag am Boden eines Okosystems. auftreffend. 
B. Begriffsfelder und jeweilige auf Exkursionen zu behandelnde Beispiele (zu andern Wissensbereichen hinführend)

Allgemeine Begriffe

Beispiele

1. Ökologische Beziehungen von Organismen untereinander

Miteinander von Oraganismen:

Symbiose im engeren Sinne, Abhängigkeitsverbindungen von Pflanzen untereinander

Flechten (Bau, Extremstandorte)

Epiphytismus

Kletterpflanzen (Morphologie)

Mikroklima im Walde (Mikroklimatologie)

Stickstoffsammler und Nitrophyten

(Ackerbau)

Bestäubungsökologie

Ausbreitungsökologie

Vikarianz, Stellenäquivalenz

Gegeneinander von Organismen:

Konkurrenz

Parasitismus, Schädlingsbefall,

1. Thienemann'sche Regel allüberall zahlr. Beispiele (Zoologie)

Dominanz von Myrmekochorie oder Ornithochorie in best. Gesellschaften (Morphologie)

Höhenvikarianten (z.B. Senecio-Arten); edaph. Vikar. (z.B. Rhododendron-Arten); Wasserstufen-Vikar. (z.B. Ranunculus-Arten)

Arrhenatherum - Trisetum-Stellenäquiv.

Pteridium-Herden u.ä. als Forstunkraut; Dominanz von Allium ursinum

Gallen

charakteristische Schadbilder (z.B. Posthornwickler)

(Nahrungskette, Biolog. Schädlingsbekämpfung)

II. Einpassung in die spezifische Umwelt, Anpassung an den Standort (Morphologie, Physiologie, Evolutionsbiologie)

Nischenbildung (i.S. von neuer Art der Nutzung eines bestimmten Lebensraums)

Lebensform
Wasserpflanzen als solche (Ableitung der von Luft abweichenden Standortsqualitäten des Wassers)

Abstufung an Grenze Wald-Freiland; Unkraut-Strategien 
Aufpassung in verschiedene Rhythmen

Besiedlung von Extremstandorten,

2. Thien'emann'sche Regel

Konstitutionelle und plasmatische

Resistenz

Ökologische Amplitude (ök. Valenz)

Indikatorart (Zeigerpflanze)
Blütezeiten in Mähwiese;

Blütezeit der Krautschicht in Laubwäldern

Schutthalde, Mauerfuge, Hochmoor o.ä.

Xerophyten verschiedenster Art

weit: z.B. Calluna vulgaris, Pinus sylv. eng: z.B. Quellflurarten, Erica tetralix

Bartflechten; Salzpflanzen; Kalkzeiger usf.

III. Die Beziehungen

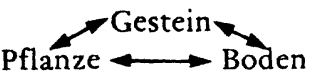

(zahlreiche Ansätze für einfache Messungen u. Faktorenanalysen)

(Geologie, Geomorphologie, Pedologie)

Pflanzen und Gesteinsbildung

Pflanzen und Bodenbildung
Kalktuffbildung, Kalkalgenbänke Moor- und Torfentstehung (Kohle) (mit Tieren: Sapropel, Erdöl)

Streu-u. Humusproduktion Sprengung von Gestein durch Wurzelwachstum

chemische Verwitterung durch Säureproduktion (z.B. endolith. Kalkflechten Kaolinisierung von Feldspat durch Flechten)

Bedeutung des Bodens für die Pflanze als Substrat zur Verankerung

als Ionen-Lieferant
Flach-u. Tiefwurzler (Sturmschaden) Fels-und Sandwatt Lebendbau an Böschungen

Pflanze als Glied im Ionenkreislauf (Trop. Regenwald)

Rohhumus-bzw. Mull-Bildung Humusgehalt in Acker, Wiese, Wald, Nieder-u. Hochmoor Mykorrhiza, Stickstoffbindung Vegetationsgrenzen zwischen Kalk- und Silikatgestein

Salzschäden in Städten 
als Wasserspeicher

als Sauerstoff-Lieferant für Wurzeln

als Nahrung für Heterotrophe
Gründigkeit und Steingehalt verschiedener Standorte, u.a. Schutt- und Spaltenstandorte

Nutzung verschiedener Stockwerke durch versch. Wurzeltiefen

Sand-, Lehm-u. Tonböden

Beobachtung von Trockenschäden

Produktionsintensität pro Flächeneinheit (Beregnung)

Grund wasserabhängigkeit von Auwäld., Wiesen auf Gleyböden

Entwässerungsfolgen

Stauhorizonte

Durchwurzelungsintensität d. Horizonte Aerenchym bei Sumpfpflanzen (biochem. Anpassung)

Pilzmycel

belebte Streu, Bodentiere

Zersetzung von Stümpfen

IV. Die Beziehungen Pflanze $\leftrightarrow$ Klima

(zahlreiche Gelegenheiten zu einfachen Messungen und Faktorenanalysen)

(Mikroklimatologie)

Strahlungshaushalt

Expositionsabhängige Vegetation an

Hängen

Dauer der Schneedecke, Schutz und Ver-

kürzung der Vegetationsperiode, Schneetälchen

Schneedruck, Lawinenbahnen, L.-verbau

Kaltluftgefährdung, Frostschadenbeo-

bachtung (Walnuß, Reben, Kartoffeln)

Ungleiche Erhitzung der Substrate (Torf

- Gestein - Bult - Schlenke)

Frosttrocknis

Licht als limitierender Faktor im Wald, in Höhlen (Profil)

Anpassung an geringen Lichtgenuß, Lia-

nen

Licht- und Schattholzarten und ihre

Konkurrenzfähigkeit 
Atmosphärischer Wasserhaushalt

Wind

Luftverunreinigung $u$. andere Immissionen
Austrocknungsgeschwindigkeit bei Moosen

Epiphytenbewuchs

Transpirationshemmung: Xeromorphe gegen Hygromorphe (Hochstauden)

Sukkulente (Morph., Biochemie)

Windformen, Weiser für Hauptwindrichtung

„Windecken"-Vegetation

„Flechtenwüste" in Städten, Kryptogamen um Emittenten

Staubschutzgehölze

Salzgischt am Meer

Lärmschutz durch Gehölze

V. Die räumliche Verteilung von Pflanzengesellschaften

(Vegetationsprofile, Veg. karten; zur Kausalität s. III und IV)

Zonation

Höhenstufung

Mosaikbildung

Landschaftscharakteristische Komplexe

Vegetationsgrenzen
Uferzonen von Seen; Dünengürtel und ihre edaphische Bedingtheit

Veget. stufen im Gebirge u. ihre klimatische Bedingtheit

Überflutungsabhängige Komplexe in Flußauen

Wirtschaftsbedingte Komplexe homologer Gesellschaften

Sandheide-Landschaft, Eichen-Hainbuchenwald-Landschaft

Allmählich, kontinuierlich, naturnah:

Wald - Mantel - Saum; abrupt, scharf, anthropogen: Parzellenund Nutzungsgrenzen Waldgrenztypen: mit einzelnen Bäumen, mit geschlossener Krüppelzone; ihre Kausalität 
VI. Die zeitliche Änderung von Pflanzengesellschaften

(Sukzcssionsforschung und Vegetationsgeschichte)

Pionicrpflanzen

Sukzessionen und ihre Stadien

Natürliche (aktuelle oder

potentielle) Vegetation

Adventivpflanzen $u$. Wanderungen Florenverfälschung

Relikte und Reliktstandorte
Frstbesic dlung von Baggersecn, von Böschungen (Ausbreitungsökologic)

Sozialbrache verschiedenen Alters auf Äckern, Wiesen, Weiden; Änderung des Lebensformspektrums

Wald als Klimax; Schlußglieder extremer Standorte

Ursprüngliche Standorte von Acker-u. Wiesenarten; Neophyten-reiche Standorte; Eigenschaften von Neophyten, die Dominanz bewirken

Eiszeitliche und wärmezeitliche Relikte; progressive $u$. regressive $R$. Charakteristik von R. standorten (Sippenbildung)

VII. Im Blickpunkt: Der wirtschaftende Mensch (Abschnitt I-VI, Landwirtschaft, Forstwirtschaft, Naturschutz)

Bereicherung und Verarmung der Natur als intensitätsabhängige Prozesse; Landschaftspflege
Struktur, Pfl. gesellschaften und Arteninventar der bäuerlich geprägten gegenüber der technisierten Landschaft Halbkultur-versus Intensivstformationen Flurbereinigung Sozialbrache standortsgemäße Ufer- und Straßenböschungsgestaltung, Landschaftswunden

ehem. Nieder- und Hudewälder Aufforstung, Naturverjüngung standortsgemäße Artenwahl Hiebsweisen (Kahl-, Schirmschlag u.ä.) Naturwaldrescrvate (Bannwälder)

Wohlfahrtswirkungen des Waldes
Erholungswald

Boden-, Wasscr-, Lawinen-, Immissionsschutzwald 
Ackerbau

Standortsabhängigkcit

Grenzen in der Landschaft

Limiticrung durch das Standortspotential landschaftsspezifisches Kulturpflanzensortiment

Standortsprägung

Reaktion der Unkrautflora auf Bewirtschaftsrhy thmus

Einfluß von Düngung, von Bioziden

Zeigerpflanzen für Pflugsohlenverdichtung, für Oberflächenverdichtung, für Nährstoffmangel

Erosion und Humusverlust und Gegenmaßnahmen

Grünlandwirtschaft

Standörtliche Limitierung, absolutes

Grünland

Typenwechsel in Abhängigkeit vom

Srandortspotential

Wasserstufen(karte)

Wirkung von Mahd und Beweidung

Einpassung in den Mährhythmus

Weidefestigkeit, Weideunkräuter

\section{Schlußwort}

Exkursionen können - auch wenn sie nur in Zivilisationslandschaften führen eine Fülle von biologischen Erlebnissen vermitteln, die sich in ein theoretisches Begriffssystem der Ökologie einbauen lassen, und dazu Verbindungen zu andern Teildisziplinen aufzeigen. Sie können damit auch die persönliche Beziehung zur Natur, die Fähigkeit zu unmittelbarer Beobachtung, zur logischen Kombination und Wunsch und Fähigkeit zu eigenem Einsatz für die Natur stärken. So möchte man den Exkursionen einen höheren Stellenwert im Kanon der Lehrverstaltungen zumessen. Denn vielen von uns scheint heute zeitgemäß, was Friedrich Nietzsche vor gut $100 \mathrm{Jahren}$ in seinen ,Unzeitgemäßen Betrachtungen“ schrieb: „Übrigens ist mir alles verhaßt, was mich bloß belehrt, ohne meine Tätigkeit zu vermehren oder unmittelbar zu beleben."

\section{Literatur}

Ellenberg, H. (1963): Vegetation Mitteleuropas mit den Alpen. 943 S. Stuttgart. Moor, M. (1962): Einführung in die Vegetationskunde der Umgebung Basels. 464 S. Basel. Osche, G. (1973): Ökologie. 143 S. Reihe: Herder/Studio visuell. Freiburg. Reichelt, G \& W. Schwoerbel, (1974): Ökologie. 64 S. (CVK-Biologie-Kolleg. Berlin.) 
Tüxen, R. (1968): Die Lüneburger Heide. Werden und Vergehen der nordwestdeutschen Heidelandschaft. (Durchgesehene und erweiterte Fassung) - In: Kelle, A. (Edit.): Neuzcitliche Biologie 9, S. 9-55. Hannover.

Wilmanns, O. (1973): Ökologische Pflanzensoziologie. 288 S. UTB Heidelberg.

Wilmanns, O. et al. (1974): Der Kaiserstuhl - Gesteine und Pflanzenwelt. 241 S. Ludwigsburg, 2. Aufl. im Druck.

Anschrift der Verfasserin:

Prof. Dr. Otti Wilmanns, Biologische Institut II der Universität, Freıburg i. Br. 